\title{
Seguimento de Instruções Não Correspondentes em Crianças de Diferentes Idades ${ }^{1}$
}

\author{
Kátia Daniele Biscouto \\ Universidade Estadual de Londrina, Londrina, Brasil \\ Andréia Schmidt* \\ Faculdade de Filosofia, Ciências e Letras de Ribeirão Preto - USP, Ribeirão Preto, Brasil
}

Dhayana Inthamoussu Veiga

Universidade Federal de São Carlos, São Carlos, Brasil

\begin{abstract}
RESUMO
A manutenção de desempenhos controlados por instruções não correspondentes pode ser atribuída a diferentes variáveis. O objetivo deste estudo foi verificar o efeito de uma história experimental de apresentação de instruções correspondentes sobre o comportamento subsequente de seguir instruções não correspondentes, em um procedimento de operante livre. Doze crianças ( 3 a 8 anos) participaram de um jogo de caça ao tesouro. As primeiras pistas do jogo (correspondentes) indicavam corretamente onde encontrar mais pistas; sem sinalização de alteração de condição, pistas não correspondentes passaram a ser apresentadas. Os participantes mantiveram-se seguindo instruções (correspondentes ou não). Gradualmente, porém, passaram a dispensar mais tempo buscando pistas em locais não indicados pelas instruções não correspondentes. Discutem-se possíveis variáveis relacionadas à manutenção do desempenho instruído.
\end{abstract}

Palavras-chave: instruções; controle por regras; operante livre; história experimental; crianças.

\section{ABSTRACT \\ Non-Correspondent Instructions Following In Children With Different Ages}

The maintenance of behavior controlled by non-correspondent instructions can be explained by a wide range of variables. The aim of the study was to verify the effect of an experimental history of correspondent instructions presentation on subsequent non-correspondent instructions-following behavior in a free-operant procedure. Twelve children from 3 to 8 years old participated in a game format task (a treasure hunt). First clues presented were correspondent (correspondently indicating where new clues were hidden); without signaling, non-correspondent instructions were presented. All participants kept following instructions (correspondent or not); however, they gradually spent more time searching for clues at places not described by non-correspondent instructions. Variables that possibly affected the maintenance of instruction-following behavior were discussed.

Keywords: instructions; rule control; free-operant procedure; experimental history; children.

Com o comportamento verbal, a esfera de ação do homem sobre seu ambiente social foi amplamente expandida devido ao caráter especial que esse tipo de comportamento operante possui: sua seleção ocorre por consequências mediadas por ouvintes especialmente treinados para esse fim, isentando-o das relações mecânicas às quais estão subordinados os demais operantes (Skinner, 1957). Poder desempenhar as funções de falante e de ouvinte possibilitou à espécie humana comportar-se além dos limites mecânicos impostos pela natureza. O fato de um ouvinte ser capaz de comportar-se em relação a uma parcela do mundo com a qual ele jamais teve contato direto, apenas por meio de sua relação com o falante, define a

* Endereço para correspondência: Andréia Schmidt - aschmidt@ffclrp.usp.br 
função global do comportamento verbal (Skinner, 1957; 1974).

O controle exercido pelo comportamento do falante sobre o comportamento do ouvinte tem sido estudado e discutido em pesquisas sobre o controle por instruções, originalmente chamado por Skinner de controle por regras (Skinner, 1966/1969). O termo instrução será utilizado no presente trabalho como sinônimo do termo "regra", definido por Skinner (1966/1969) como um estímulo discriminativo verbal que descreve uma ou mais contingências de reforçamento, ou seja, uma relação de dependência entre eventos antecedentes ao responder de um organismo, o responder e suas consequências.

O controle por instruções passou a ser investigado na Análise Experimental do Comportamento em estudos que visavam compreender diferenças observadas entre o desempenho de humanos e não humanos em diferentes esquemas de reforçamento (e.g., Lowe, Harzem, \& Bagshaw, 1978; Weiner, 1964; 1969) e em estudos especialmente conduzidos para investigar o controle instrucional (Weiner, 1970). A principal premissa era de que a presença de repertório verbal em humanos poderia propiciar a formação de autoinstruções, que passariam a exercer controle sobre o desempenho não verbal. Dessa forma, o controle instrucional tornaria o responder menos controlado pelas contingências não verbais vigentes.

Com base nessa interpretação, diversos estudos foram realizados, em sua maioria com participantes adultos, para investigar o comportamento de seguir instruções em condições experimentais, ora correspondentes, ora não correspondentes às instruções apresentadas. Padrões de desempenho inalterados diante de mudanças nas contingências de reforçamento foram chamados de "insensibilidade comportamental", considerada pelos autores um produto do controle instrucional (e.g., Catania, Matthews, \& Shimoff, 1982; Catania, Shimoff, \& Matthews 1989; Hayes, Brownstein, Zettle, Rosenfarb, \& Korn, 1986; Matthews, Shimoff, Catania, \& Sagvolden 1977; Shimoff, Catania, \& Matthews, 1981; Shimoff, Matthews, \& Catania, 1986).

Estudos mais recentes, porém, sugerem que padrões inalterados de comportamento diante de mudanças nas condições experimentais devem ser compre- endidos considerando um conjunto mais amplo de variáveis responsáveis por sua manutenção (Albuquerque, 2005; Madden, Chase, \& Joyce, 1998; Paracampo \& Albuquerque, 2005): a natureza das consequências contingentes ao seguimento ou não das instruções (Paracampo \& Albuquerque, 2004); a presença ou ausência do experimentador na sessão (Albuquerque, Paracampo, \& Albuquerque, 2004; KrogerCosta \& Abreu-Rodrigues, 2012); a história prévia de reforçamento (Albuquerque, de Souza, Matos, \& Paracampo, 2003; Albuquerque, Matos, de Souza, \& Paracampo, 2004); a duração da exposição às condições experimentais (Albuquerque, Reis, \& Paracampo, 2008); a variabilidade do responder controlado por instruções (Joyce \& Chase, 1990); e o esquema de reforçamento em vigor nas condições experimentais (Madden et al., 1998). As conclusões desses estudos e considerações feitas por outros pesquisadores (Nico, 1999; Thomaz \& Nico, 2007) levam a questionar a adequação do uso do termo "insensibilidade às contingências" (ou "pseudosensibilidade", utilizado por Shimoff et al., 1986) e, principalmente, questionam se tais padrões de resposta podem de fato ser interpretados como parte inerente ao controle instrucional.

Autores como Joyce e Chase (1990), Madden et al. (1998), Paracampo e Albuquerque (2005), Nico (1999) e Thomaz e Nico (2007) defendem que o responder controlado por instruções é um comportamento multideterminado, sujeito a diversas fontes de controle que podem ou não ter sido previstas em um experimento. Por isso, é preciso considerar aspectos de procedimento que possam funcionar como variáveis estranhas às manipulações previstas e por em questão os resultados dos estudos (Teixeira Júnior, 2009; Teixeira Júnior \& Paracampo, 2010; Teixeira Júnior \& Paracampo, 2012). Além disso, também é preciso observar que grande parte dos estudos sobre "insensibilidade às contingências" foi conduzida com participantes adultos, os quais possuem um amplo repertório de seguimento de instruções (estudantes universitários) e que os procedimentos empregados utilizam tentativas discretas, nas quais o seguimento de instruções é observado em períodos isolados de tempo e em tarefas que oferecem poucas possibilidades de emissão de respostas diferentes daquelas instruídas, sendo essas características geralmente impostas pelo próprio aparato experimental utilizado (Perone, 1991; Velasco, Garcia-Mijares, \& 
Tomanari, 2010). Embora esse tipo de procedimento se destaque pelo rigor no controle experimental, as características da tarefa, da situação experimental e o perfil dos participantes parecem interferir na investigação do fenômeno de interesse (Veiga, Schmidt, \& Biscouto, 2012).

Veiga et al. (2012) conduziram um estudo no qual se utilizou um procedimento de operante livre com crianças de 8 a 10 anos, nas dependências de uma escola de ensino fundamental. As crianças participaram individualmente de uma tarefa em formato de jogo ("caça ao tesouro") em uma única sessão, na qual se buscavam pistas que indicavam ou não locais com novas pistas, até que se encontrasse um vale brinde ("tesouro"). O objetivo era investigar se a correspondência ou não correspondência de instruções em relação às condições de reforço alteraria a função dessas instruções sobre o responder de crianças. Foram apresentados dois tipos de condição de instrução: instruções correspondentes e instruções não correspondentes. Para metade dos participantes eram predominantemente apresentadas instruções correspondentes às contingências de reforço vigentes; para os demais eram apresentadas predominantemente instruções não correspondentes. Todas as crianças seguiram instruções até o fim da sessão, embora crianças mais expostas à condição de não correspondência tenham emitido mais comportamentos com topografia diferente da instruída. Veiga et al. (2012) interpretaram que instruções correspondentes e não correspondentes alteraram a função dos estímulos presentes no ambiente experimental, tornando-os discriminativos para respostas de busca (Schlinger \& Blakely, 1987; Schlinger, 1993). Isto é, quando uma instrução era lida pelo participante, respostas de olhar em direção a todo o ambiente experimental eram diretamente evocadas, contudo, as respostas de busca eram evocadas pelos próprios objetos e locais descritos pela instrução lida (Veiga et al., 2012).

O estudo de Veiga et al. (2012) forneceu indicações de que, em um procedimento de operante livre, o comportamento instruído pode se manter mesmo quando as condições de instrução não são correspondentes, mas também sugeriu que, em condições de não correspondência entre instruções e contingências não verbais, pode ocorrer o aumento da probabilidade de emissão de respostas alternativas àquelas instruídas, ao menos sob as contingências do estudo realizado. Diferentemente dos procedimentos por tentativas discretas tradicionalmente adotados, o procedimento de operante livre utilizado por Veiga et al. permitia que os participantes emitissem uma variedade muito maior de respostas (funcionalmente e topograficamente variadas) a qualquer momento da sessão, em relação a quaisquer objetos disponíveis no ambiente, sem que houvesse restrições sobre seu responder impostas por aparatos utilizados na tarefa experimental (Perone, 1991; Velasco et al., 2010). Os participantes eram expostos a mais oportunidades de se engajarem em respostas que não tivessem relação com a tarefa experimental, como brincar isoladamente com os objetos disponíveis no ambiente ao invés de buscarem por pistas. Em um procedimento por tentativas discretas essa possibilidade costuma ser minimizada ou restrita ao uso de um tipo determinado de aparato, como um computador. Nesse caso, o participante tende a se engajar exclusivamente nas respostas exigidas pela tarefa (clicar com o mouse, pressionar teclas, tocar a tela) e a permanecer sentado diante do aparato durante todo o procedimento. Esse aspecto de procedimento pode ser especialmente relevante ao se estudar o desempenho de crianças.

Embora estudos sobre controle por instruções conduzidos com crianças sejam menos frequentes, tem-se analisado diferentes variáveis que podem interferir na manutenção do comportamento instruído em condições de correspondência ou não com as contingências não verbais. Baxter e Schlinger (1990) e Bentall, Lowe e Beatsy (1985) investigaram o efeito de diferentes níveis de desenvolvimento e repertório verbal sobre o desempenho de crianças em tarefas operantes sob diferentes esquemas de reforçamento, buscando semelhanças ou diferenças entre esse desempenho e o tipicamente apresentado por não humanos. Bentall et al. (1985), por exemplo, estudaram o ajustamento do desempenho de crianças de diferentes faixas etárias em uma tarefa sob esquema de intervalo fixo (FI), que variou de 10 a 70 segundos entre sessões. Nesse estudo, grupos de crianças (as mais jovens entre 2 anos e meio e 4 anos, e as mais velhas, entre 5 e 9 anos) foram submetidas a uma tarefa em que poderiam produzir reforçadores a partir da operação de um equipamento que funcionava em esquema de FI. De acordo com os dados, enquanto as crianças mais velhas, entre 
7 e 9 anos, apresentaram um padrão de responder semelhante ao de adultos (não ajustado às contingências em vigor), as crianças mais jovens, entre 2 anos e meio e 4 anos, apresentaram curvas de desempenho semelhantes às de animais não humanos. As crianças de 5 e 6 anos mostraram um padrão mais variável, aparentemente intermediário entre os outros dois grupos. Os autores sugeriram que repertórios verbais mais elaborados permitem a formulação de autoregras, que tendem a controlar o desempenho e a se manter mesmo com as mudanças nas contingências; por outro lado, crianças mais novas, por não possuírem repertório verbal suficiente para elaborar tal descrição, tendem a ficar mais sob controle das contingências em vigor.

Variáveis de outra natureza têm sido investigadas por outros pesquisadores. Paracampo, de Souza e Albuquerque (2001) investigaram a interferência de diferentes formas de estabelecimento de desempenho em uma tarefa de matching to sample sobre a manutenção desse desempenho após mudanças não sinalizadas de condições de reforço, em crianças de 7 e 8 anos; Michael e Bernstein (1991) também investigaram a interferência da forma de estabelecimento de um desempenho (por instruções, reforço diferencial ou imitação) na aprendizagem de discriminações condicionais e formação de classes de equivalência sobre a aprendizagem da reversão de algumas das relações ensinadas, em crianças de 4 e 5 anos; Paracampo e Albuquerque (2004) investigaram o papel das consequências na manutenção de comportamento instruído em contingências de reforço discrepantes das regras, em crianças de 7 a 9 anos. Embora tais estudos tenham sido conduzidos com diferentes objetivos, sem que a idade dos participantes fosse uma variável experimental, os dados gerados com crianças de diferentes faixas etárias permitem uma leitura geral desse conjunto de artigos, que sustenta a justificativa do presente trabalho. A maioria dos estudos demonstra uma tendência das crianças mais velhas manterem o desempenho instruído quando as contingências de reforço passam a ser não correspondentes, exceto em condições de perda de reforçadores (Paracampo \& Albuquerque, 2004) ou em condições combinadas de desempenho adquirido por instruções e reforçamento diferencial (Monteles, Paracampo, \& Albuquerque, 2006). Porém, o mesmo não ocorreu no estudo de
Michael e Bernstein (1991) com pré-escolares, no qual foi observada uma mudança no padrão de resposta que acompanhava as mudanças nas contingências para todos os participantes, independente da forma como o desempenho foi adquirido. Essa diferença entre o desempenho de crianças mais novas e mais velhas nos diferentes estudos sugere a necessidade de novas investigações que comparem diretamente o desempenho de crianças de diferentes faixas etárias, de modo a identificar condições que favoreçam ou dificultem mudanças no desempenho instruído em função de mudanças nas contingências de reforçamento.

A proposta do presente estudo foi realizar uma análise do controle por instruções com crianças de diferentes faixas etárias utilizando um procedimento de operante livre adaptado de Veiga et al. (2012). O objetivo do trabalho foi verificar o efeito de uma história experimental de apresentação de regras correspondentes sobre o comportamento subsequente de seguir instruções não correspondentes em uma situação de operante livre, comparando os desempenhos de participantes de diferentes faixas etárias $(3,5$ e 8 anos).

\section{MÉTODO}

\section{Participantes}

Participaram individualmente da pesquisa 12 crianças, distribuídas em três grupos de acordo com sua faixa etária: duas meninas e dois meninos de 8 anos; três meninas e um menino de 5 anos; três meninas e um menino de 3 anos. A participação das crianças foi autorizada pelos pais mediante assinatura de Termo de Consentimento Livre e Esclarecido.

\section{Materiais e Equipamentos}

A sessão experimental era conduzida em uma sala de aproximadamente $28 \mathrm{~m}^{2}$, equipada com espelho unidirecional. Foram distribuídas pela sala 17 caixas de papelão $(36 \times 12 \times 26 \mathrm{~cm})$ que continham diferentes materiais (e.g., bolas de isopor de tamanhos diferentes, papel colorido picado, petecas, garrafas plásticas de $600 \mathrm{ml}$ vazias, cordas de sisal, bonecos de pano, pedaços de papel amassados, sucatas, novelos de lã de cores variadas e revistas, etc). Essas caixas foram denominadas "locais de busca", onde as crianças pro- 
curavam pequenos blocos de madeira que seriam trocados por novas pistas. Em cada caixa, havia um único tipo de material. Outros locais de busca foram uma lata de metal com tampa, um túnel de pano de aproximadamente $1,50 \mathrm{~m}$, três almofadas de pano medindo $45 \times 45 \mathrm{~cm}$, uma pilha de toalhas de banho e três cadeiras. Na sala também havia um palco para teatro de fantoches e um fantoche de mão, que apresentava verbalmente as pistas para as crianças. Foi utilizada uma caixinha de presente dourada $(6 \times 6 \times 3 \mathrm{~cm})$ como "tesouro". Ela deveria ser encontrada ao final do procedimento e trocada por um brinde (artigos de papelaria como, por exemplo, colas coloridas ou fitas adesivas coloridas). Todo o procedimento foi filmado com uma câmera (Panasonic, VHS-M9000).

\section{Situação experimental}

As crianças participaram de uma brincadeira chamada "Caça ao Tesouro", cujo objetivo era encontrar um "tesouro" que estava escondido em algum lugar da sala. Para isso, os participantes tiveram que seguir pistas, apresentadas verbalmente por um fantoche manipulado pela pesquisadora. Cada pista apresentada pelo fantoche levava a um local de busca. Nesse local, a criança deveria procurar por um pequeno bloco de madeira azul ("bloquinho"). Ao encontrá-lo, deveria entregá-lo para o fantoche, que apresentaria uma nova pista, e assim sucessivamente, até que a última pista indicasse o local onde o tesouro ("caixinha dourada") poderia ser encontrado.

Os blocos de madeira foram distribuídos nos diferentes locais de busca presentes na sala. Para crianças de 3 anos, havia 13 locais de busca, e em oito deles havia um bloquinho; para as crianças de 5 e 8 anos, havia 22 locais de busca, 14 deles contendo um bloquinho. A caixinha dourada permanecia embaixo de três almofadas, localizadas no chão em um dos cantos da sala.

O jogo era composto por oito pistas para crianças de 3 anos e por 14 pistas para crianças de 5 e 8 anos. $\mathrm{O}$ número reduzido de pistas e de locais de busca para as crianças de 3 anos foi implementado devido a um experimento piloto, que mostrou que o engajamento das crianças menores na tarefa era melhor em um procedimento mais curto, com menos opções de busca. Metade das pistas apresentadas era corresponden- te, isto é, indicavam um local de busca onde um bloquinho seria efetivamente encontrado: Pistas 1 a 7 para crianças de 8 e 5 anos e Pistas 1 a 4 para as crianças de 3 anos; as demais pistas eram não correspondentes, indicando, portanto, locais de busca onde não havia qualquer bloquinho: Pistas 8 a 14 para as crianças de 8 e 5 anos e Pistas 5 a 8 para as crianças de 3 anos. Uma última pista correspondente era apresentada para todos os participantes ao final do procedimento, indicando a localização do tesouro ("Procure o tesouro embaixo das almofadas"): Pista 15 para as crianças mais velhas e Pista 9 para as crianças de 3 anos.

O jogo começava com a apresentação de uma sequência de pistas correspondentes (quatro pistas correspondentes para as crianças de 3 anos e sete para as crianças de 5 e 8 anos). Em seguida, sem qualquer sinalização, havia a reversão das condições de instrução, ou seja, uma sequência de pistas não correspondentes passava a ser apresentada (quatro instruções para as crianças de 3 anos e sete para os demais participantes). O jogo se encerrava quando a criança encontrava a caixinha dourada e a trocava por um brinde à sua escolha.

\section{Procedimentos}

O estudo foi aprovado pelo Comitê de Ética em Pesquisa da Universidade Positivo (Protocolo $\mathrm{n}^{\circ}$ 48/2009). Inicialmente, uma auxiliar de pesquisa convidava a criança para conhecer a sala, passando por todos os locais de busca e perguntando o nome que a criança dava para os materiais espalhados pelo chão da sala. Esses nomes eram anotados pela pesquisadora, que os empregaria na descrição das pistas. Depois desse reconhecimento do local, era lida para o participante a instrução inicial do jogo:

Aqui nessa sala tem um tesouro escondido. O tesouro é igualzinho a esta caixa (era apresentada a caixinha dourada). Só que dentro desta caixa não tem nada. Quando você achar essa caixinha, você vai poder trocar esse tesouro por um desses presentes (era apresentado um mostruário com os brindes). Olha quantas coisas tem aqui dentro. Vamos ver o que tem? (os brindes eram apresentados para a criança) Quando você achar o tesouro você vai poder trocar por um desses brinquedos aqui, mas só no final (o 
mostruário era retirado do campo visual da criança). Como é que a gente vai fazer para achar o tesouro? Aqui tem um fantoche. O nome dela é Nica. Vamos conversar um pouco com a Nica? (Nesse momento, o fantoche interagia com a criança, perguntando seu nome e convidando-a para brincar) A Nica é que vai contar o que você tem que fazer para achar o tesouro. Ela vai te dizer muitas coisas que você vai ter que fazer para achar o tesouro. Para achar o tesouro, você vai ter que trazer para a Nica uma porção de bloquinhos de madeira como esses aqui (dois blocos eram apresentados). Esses bloquinhos estão escondidos aqui nessa sala, e podem estar em qualquer lugar. A Nica vai te dizer onde procurar. Ela vai te dizer dentre todos aqueles lugares ali, onde você vai procurar esses bloquinhos (os bloquinhos eram apresentados novamente). A Nica vai dizer: $X$, vá procurar embaixo da cadeira, e aí você vai procurar e vai trazer para Nica um desses aqui. Cada vez que você trouxer um desses a Nica vai te dizer outra coisa que você tem que fazer. Agora vamos fazer uma vez? (era dado um bloquinho para a criança levar para o fantoche, que pedia para a criança procurar embaixo da cadeira; esperava-se a criança achar a peça, entregar ao fantoche e comemorava-se com ela). Entendeu? Agora vamos começar de verdade a brincadeira? Eu vou sentar aqui e ficar te olhando brincar.

Caso a criança dissesse que não havia entendido, ou se, no início do procedimento, a criança não atendesse à primeira instrução do fantoche, a auxiliar de pesquisa repetia a instrução inicial. Para iniciar o procedimento, a auxiliar de pesquisa levava a criança até o fantoche e o jogo era iniciado, assim como o registro do tempo da sessão. Inicialmente, era apresentada uma sequência de pistas correspondentes. Em seguida, era apresentada uma sequência de pistas não correspondentes. Apesar dos bloquinhos de madeira ficarem dentro dos locais de busca misturados a diferentes materiais, eles estavam à vista das crianças, podendo ser localizados com facilidade. Esse recurso foi utilizado para verificar se, ao não encontrar o bloquinho a partir de uma pista não correspondente, a criança abandonaria a busca no local indicado (deixando de seguir a instrução não correspondente) e se engajaria na procura do bloquinho em outros locais de busca em que probabilidade de sucesso era aumentada. Os locais de busca indicados pelas instruções não correspondentes sempre eram próximos de locais de busca onde efetivamente um bloquinho poderia ser encontrado. Por exemplo, se a pista não correspondente era "Procure o bloquinho na caixa com cordas", ao lado da caixa com cordas (onde não havia qualquer bloquinho) havia uma caixa com argolas que continha um bloco de madeira. O jogo se encerrava com a apresentação da pista que indicava corretamente a localização do tesouro. Caso o participante encontrasse a caixinha dourada antes de todas as pistas serem apresentadas, a sessão era encerrada com a troca do tesouro por um brinde.

Caso um participante permanecesse em um mesmo local de busca falso (sem um bloco de madeira) por dez (crianças de 8 anos) ou cinco minutos (5 e 3 anos) consecutivos, a auxiliar de pesquisa apresentava uma pista adicional para facilitar a exploração do ambiente: "os blocos podem estar escondidos em qualquer lugar dessa sala". Esse procedimento foi adotado para prevenir a ocorrência de respostas emocionais das crianças, após a reversão das condições de instrução.

\section{Procedimento de Análise de Dados}

Foram registradas a topografia e a duração das respostas de busca, no intervalo entre a apresentação da pista e a criança tocar no bloco de madeira encontrado. As respostas dos participantes foram categorizadas como "Respostas de seguimento de pista atual", "Respostas de seguimento de pista anterior", "Respostas de exploração do ambiente" e "Outras". "Respostas de seguimento de pista atual" envolviam o contato com um ou mais objetos descritos na última pista apresentada. Por exemplo, se a pista era "Procure na caixa com argolas", eram consideradas respostas de seguimento de pista atual andar em direção à caixa de argolas, tocar a caixa de argolas, revirar seu conteúdo, etc. "Respostas de seguimento de pista anterior" eram aquelas que envolviam o contato com um ou mais objetos descritos em pistas que já haviam sido apresentadas anteriormente à última pista. "Respostas de exploração do ambiente" eram aquelas que envolviam o contato com objetos ou locais que não haviam sido indicados em quaisquer pistas apresentadas. Foram classificadas como "Outras" respostas que não se enquadravam nas categorias descritas, tais como andar com as mãos no bolso da blusa, permanecer parado encostado na parede, permanecer olhando para a fil- 
madora, fantoche ou auxiliar de pesquisa, arrumar as roupas ou remexê-las, etc.

Após o registro e categorização das respostas para cada pista, foi calculada a porcentagem de tempo de ocorrência de cada uma das categorias.

\section{RESULTADOS}

Os dados dos participantes individuais estão representados separadamente, em três figuras, de acordo com a faixa etária. São apresentadas as porcentagens de tempo dispensado na emissão de cada uma das categorias registradas (em cada pista): "Respostas de seguimento de pista atual" (barras pretas), "Respostas de exploração do ambiente" (barras cinza escuras), "Respostas de seguimento de pista anterior" (barras cinza claras) e "Outras" (barras brancas). Quando última pista foi encontrada (que indicava o local do tesouro), todos os participantes passaram $100 \%$ do tempo engajados em seguimento de pista atual. Desta forma, o tempo de engajamento diante dessa pista foi omitido nas figuras.

A Figura 1 apresenta os desempenhos individuais dos participantes de 8 anos de idade (C8a, C8b, C8c, C8d) diante de cada uma das pistas recebidas durante o procedimento.
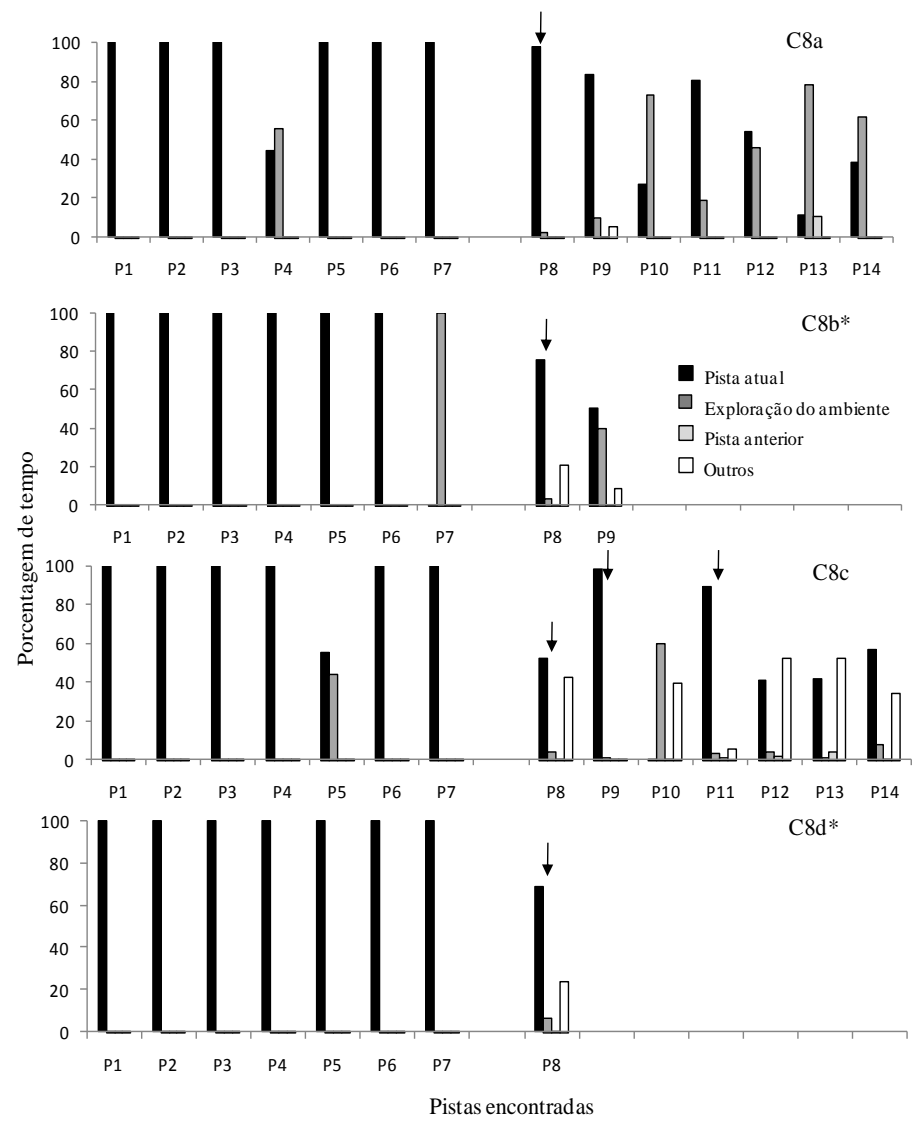

Figura 1. Porcentagem de tempo dispensado na emissão de respostas de seguimento de pista atual (preto), exploração do ambiente (cinza escuro), pista anterior (cinza claro) e outros comportamentos (branco), dos participantes de 8 anos (C8a, C8b, C8c e C8d). 0 espaço entre as pistas P7 e P8 indica mudança nas condições de instrução (de correspondentes para não correspondentes). As setas indicam o momento de apresentação de pistas adicionais. Asteriscos indicam crianças que encontraram o tesouro antes da última pista (ver texto).

Nas pistas correspondentes (P1 a P7), de modo geral, todos os participantes de 8 anos passaram $100 \%$ do tempo engajados no seguimento de pista atual. Os participantes C8a e C8c nas Pistas 4 e 5, respectiva- 
mente, iniciaram a busca emitindo respostas de exploração de ambiente aparentemente sob o controle de um local não especificado pela instrução. Ao se depararem com um local sem o bloquinho, dirigiram-se para o local de busca de fato indicado pela instrução e emitiram respostas de seguimento de pista atual (com isso, nessas pistas, C8a e C8c passaram aproximadamente $50 \%$ do tempo em cada categoria de resposta). Diante da Pista 7, C8b também parece ter ficado sob o controle de um local diferente do indicado pela pista, passando $100 \%$ do tempo engajado em exploração do ambiente. No caso desse participante (C8b), a exploração do ambiente foi realizada em um local onde havia um bloquinho de madeira, o qual era diferente daquele que a pista atual indicava.

Em todas as pistas não correspondentes recebidas, a resposta emitida inicialmente por todos os participantes foi seguimento de pista atual. Diante da primeira pista não correspondente ( $\mathrm{P} 8)$, todos passaram no mínimo $50 \%$ do tempo engajados no seguimento de pista atual (mais de $70 \%$ considerando $\mathrm{C} 8 \mathrm{a}, \mathrm{C} 8 \mathrm{~b}$ e C8d). Todos, no entanto, passaram a explorar o ambiente depois de apresentada uma pista adicional.

Nas pistas não correspondentes subsequentes (P8 a P14), C8a e C8c continuaram a se engajar em respostas sob controle de pista atual, mas a porcentagem de tempo gasto nessa categoria de respostas diminuiu ao longo das pistas: de $97 \%$ do tempo na Pista 8 para $10 \%$ e $38 \%$ do tempo nas Pistas 13 e 14, respectivamente, para C8a; e $41 \%$ e $57 \%$ do tempo engajado em seguimento de pista atual nas Pistas 13 e 14, respectivamente, para C8c. Além disso, o tempo dispensado na exploração do ambiente para C8a aumentou ao longo das pistas não correspondentes: de $2 \%$ do tempo na Pista 8 , para $78 \%$ e $61 \%$ do tempo nas pistas 13 e 14 , respectivamente. $\mathrm{C} 8 \mathrm{c}$, por sua vez, após todas as pistas não correspondentes, iniciava sua busca seguindo a pista atual. No entanto, ao deparar-se com um local onde não havia bloquinho, passava a emitir "outros comportamentos", que não estavam diretamente relacionados ao jogo (permanecia, em geral, parado, olhando em direção à auxiliar de pesquisa). Em três pistas não correspondentes ( $\mathrm{P} 8, \mathrm{P} 9$ e $\mathrm{P} 10)$, foram apresentadas pistas adicionais até que $\mathrm{C} 8 \mathrm{c}$ passou a explorar o ambiente.

Os participantes C8b e C8d também se engajaram inicialmente em respostas de seguimento de pista atual na primeira pista não correspondente (P8 - 75 e $69 \%$ do tempo, respectivamente), mas ao se engajarem na exploração do ambiente após não encontrarem o bloquinho no local indicado pela pista, encontraram o "tesouro" antes da exposição a todas as pistas programadas: na P8 para C8d e na P9 para C8b.

Em média, os participantes de 8 anos se engajaram no seguimento de pista atual em $61 \%$ do tempo, em exploração do ambiente em $21 \%$ do tempo e em outros comportamentos em $18 \%$ do tempo. Não foram observadas respostas de engajamento em pista anterior entre os participantes desse grupo.

A Figura 2 apresenta os desempenhos individuais dos participantes de 5 anos de idade (C5e, C5f, C5g e $\mathrm{C} 5 \mathrm{~h}$ ) diante de cada uma das pistas recebidas durante o procedimento. 


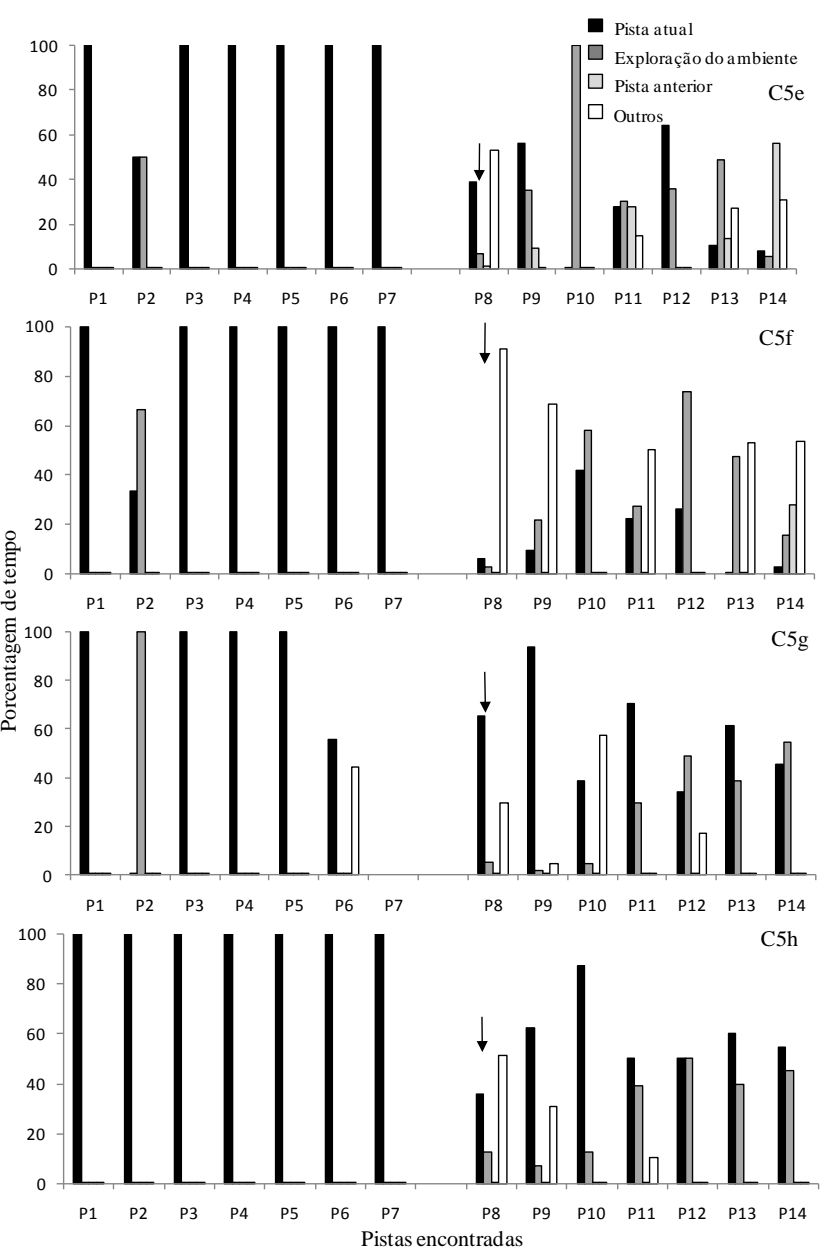

Figura 2. Porcentagem de tempo despendido na emissão de respostas de seguimento de pista atual (barras pretas), exploração do ambiente (barras cinza escuras), seguimento de pista anterior (barras cinza claras), e outros comportamentos (barras brancas), dos participantes de 5 anos (C5e, C5f, C5g e C5h). 0 espaço entre as pistas P7 e P8 indica mudança nas condições de instrução (de correspondentes para não correspondentes). As setas indicam o momento em que houve apresentação de pistas adicionais.

Assim como os participantes de 8 anos, os de 5 engajaram-se exclusivamente no seguimento de pista atual (100\% do tempo) na maioria das instruções correspondentes (P1 a P7), com exceção de C5e, C5f e C5g na Pista 2. Para esses participantes, a instrução ("Olhe a caixa com papeis coloridos") evocou a busca em lugares diferentes, o que sugere que ela foi pouco clara ou que indicou locais semelhantes entre si, do ponto de vista das crianças dessa idade. Isso parece ter dado margem a interpretações equivocadas acerca do local instruído, gerando a distribuição do responder entre seguimento de pista atual (respectivamente $40 \%$, $35 \%$ e $0 \%$ do tempo) e exploração do ambiente (50\%, $65 \%$ e $100 \%$ do tempo). O participante C5g emitiu busca em um local não indicado pela Pista 2 (caixa com revistas), mas que continha um bloquinho de madeira. Esse local seria descrito na Pista 7. Considerando o ocorrido, a Pista 7 não foi apresentada para esse participante, que também apresentou "outros comportamentos" na Pista 6 ( $44 \%$ de tempo), além do engajamento no seguimento de pista atual (66\% do tempo).

Pode-se observar, ainda na Figura 2, que todos os participantes dispensaram alguma porcentagem de tempo no seguimento de pista atual em todas as pistas não correspondentes (P8 a P14). De modo geral, observa-se que na P8 a porcentagem de tempo de emissão de outros comportamentos foi maior que a de seguimento de pista atual para C5e, C5f e C5h (52\%, $91 \%$ e $51 \%$ de tempo em outros comportamentos, em 
comparação a 39\%, 6\% e 35\% de tempo em seguimento de pista atual). Esses três participantes inicialmente seguiram pista atual e passaram a emitir outros comportamentos diante da ausência do bloquinho de madeira. A partir da apresentação da pista adicional, passaram a explorar o ambiente.

Nas pistas não correspondentes seguintes, observa-se inicialmente um aumento da porcentagem de tempo gasto em seguimento da pista atual (persistência na busca do bloquinho indicado pela pista não correspondente), seguido de uma diminuição desse tempo nas últimas pistas. Além disso, observa-se que os participantes $\mathrm{C} 5 \mathrm{e}, \mathrm{C} 5 \mathrm{~g}$ e $\mathrm{C} 5 \mathrm{~h}$ deixaram de emitir "outros comportamentos" nas últimas pistas. C5f, no entanto, continuou emitindo "outros comportamentos" nas duas últimas pistas (52\% e 53\% do tempo), assim como "exploração do ambiente" (47\% e $15 \%$ do tempo). Esse participante foi o que menos se engajou em seguimento de pista atual nas duas últimas pistas $(0 \%$ na Pista 13 e $2 \%$ de tempo na Pista 14). Em média, os participantes de 5 anos se engajaram no seguimento de pista atual em $40 \%$ do tempo, em exploração do ambiente em $32 \%$ do tempo e em outros comportamentos e $23 \%$ do tempo (o tempo restante foi computado como seguimento de pista anterior).

Na Figura 3 pode-se observar a porcentagem de tempo dispensada pelos participantes individuais de 3 anos de idade (C3i, C3j, C31 e C3m) em cada categoria de resposta registrada, diante de cada uma das pistas recebidas durante o procedimento.
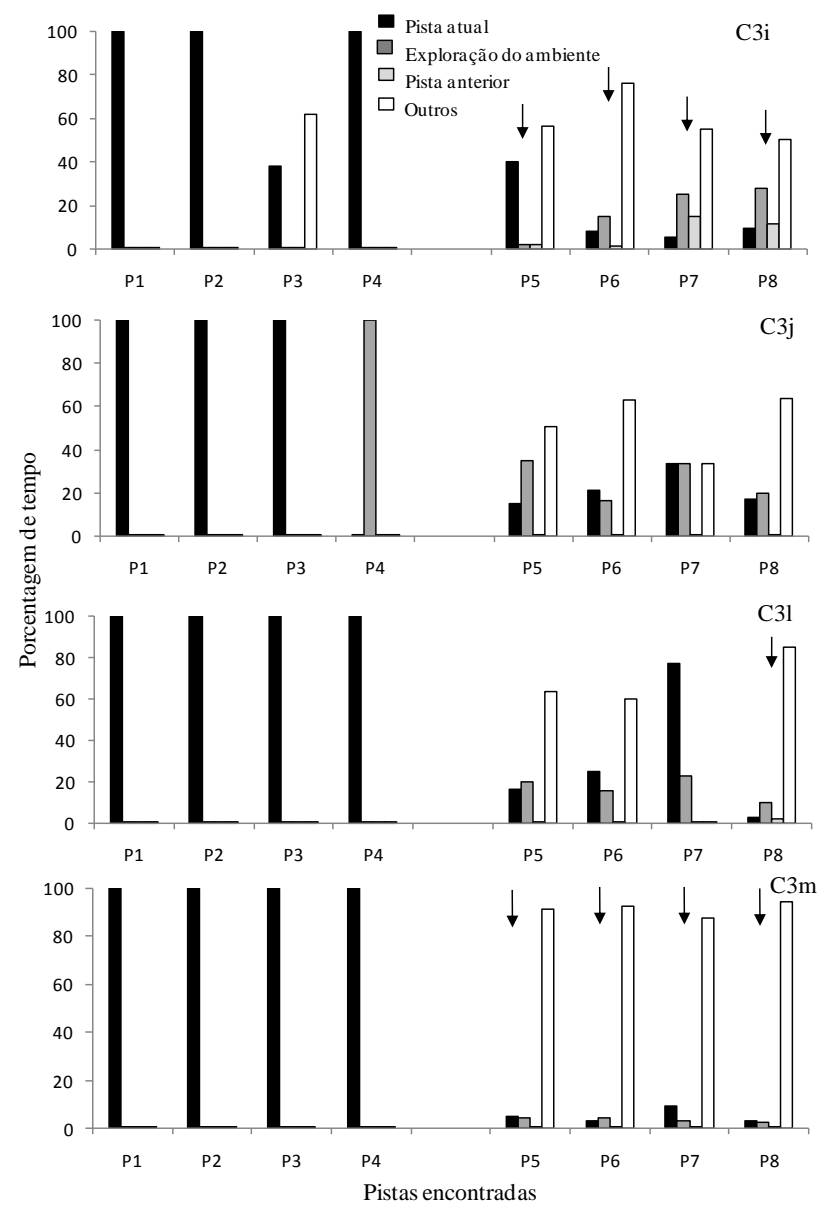

Figura 3. Porcentagem de tempo despendido na emissão de respostas de seguimento de pista atual (barras pretas), exploração do ambiente (barras cinza escuras), seguimento de pista anterior (barras cinza claras), e outros comportamentos (barras brancas), dos participantes de 3 anos (C3i, C3j, C3I e C3m). O espaço entre as pistas P4 e P5 indica mudança nas condições de instrução (de correspondentes para não correspondentes). As setas indicam o momento em que houve apresentação de pistas adicionais. 
Observa-se que todos passaram $100 \%$ do tempo seguindo pista atual em todas as pistas correspondentes apresentadas, com exceção C3i na Pista 3 e de C3j na Pista 4. Esses dois participantes pareceram ficar sob o controle de locais diferentes dos indicados pelas pistas em vigor, o que gerou uma distribuição do responder entre o local indicado pela pista e outros locais de busca disponíveis (exploração do ambiente). Aparentemente, $\mathrm{C} 3 \mathrm{i}$ e $\mathrm{C} 3 \mathrm{j}$ apresentaram respostas semelhantes às descritas para os participantes C5e e C5f na Pista 2.

Com a mudança das condições de instrução (de correspondente para não correspondente), todas as crianças mantiveram-se procurando os bloquinhos nos locais indicados pela pista atual. No entanto, em comparação com os participantes de outras idades, a porcentagem de tempo dispensada pelas crianças de 3 anos em seguimento de pista atual foi menor: em média 15\% (C3i), 21\% (C3j), 30\% (C3l) e 5\% (C3m) nas quatro pistas não correspondentes. Em contraste, essas crianças apresentaram, em média, a maior porcentagem de tempo e emissão de outros comportamentos (permanecer parado, olhando em direção ao fantoche ou à auxiliar de pesquisa, eventualmente falando frases como "Não tem bloco aqui", ou "E agora, o que eu faço?”): $59 \%$ (C3i), 52\% (C3j), 52\% (C31) e $91 \%$ $(\mathrm{C} 3 \mathrm{~m})$ do tempo, em média, no conjunto das quatro instruções não correspondentes.

Além disso, duas crianças (C3i e $\mathrm{C} 3 \mathrm{~m})$ necessitaram da apresentação de pista adicional após todas as quatro pistas não correspondentes para concluir $o$ procedimento. $\mathrm{C} 3 \mathrm{~m}$, em particular, dirigia-se aos locais de busca indicados pela pista e, após rápida inspeção e constatação de ausência do bloco, engajava-se em outros comportamentos pelo tempo limite para apresentação de pista adicional; após essa pista, rapidamente ela encontrava um bloquinho em outro local de busca. Em função disso, a porcentagem de tempo engajamento dessa criança em seguimento de pista atual foi baixa por conta da baixa persistência no seguimento de pista atual e alto engajamento em outros comportamentos. Mas, deve-se considerar que, em todas as pistas não correspondentes, o seguimento de pista atual foi a primeira resposta emitida pela criança. As outras duas (C3j e C3l), por outro lado, diante da primeira pista não correspondente, passaram a explorar os demais locais de busca (além de emitirem ou- tros comportamentos) e permaneceram se comportando dessa forma diante de todas as demais instruções não correspondentes. Foi apresentada uma pista adicional (repetição da última pista) à participante $\mathrm{C} 31$ apenas no final do procedimento. Em média, os participantes de 3 anos dispensaram 17\% do tempo em seguindo de pista atual, $15 \%$ em exploração do ambiente e $63 \%$ do tempo emitindo outros comportamentos.

\section{DISCUSSÃO}

O presente estudo teve por objetivo verificar o efeito de uma história experimental de apresentação de regras correspondentes sobre o comportamento subsequente de seguir instruções não correspondentes em uma situação de operante livre, comparando os desempenhos de participantes de diferentes faixas etárias ( 3,5 e 8 anos).

De modo geral, observou-se que quando as pistas eram correspondentes, todos os participantes engajaram-se quase que exclusivamente no seguimento das instruções apresentadas (seguimento de pista atual), o que indica que seguir instruções era um comportamento bem estabelecido para todos, independente da idade (Catania, 1999). Na mudança das condições de instrução (de correspondentes para não correspondentes), observou-se que todas as crianças, independente da idade, continuaram seguindo as pistas atuais, o que confirma dados da literatura sobre controle por instruções obtidos com crianças (Paracampo \& Albuquerque, 2004; Paracampo et al., 2001; Veiga et al., 2012). No entanto, foram verificadas diferenças no padrão de engajamento dos participantes no seguimento desse tipo de pista e na distribuição de suas respostas em outras alternativas disponíveis, de acordo com a faixa etária.

Observou-se que, quanto mais velhas as crianças, maior a porcentagem média de tempo dispensada no seguimento de pista atual na condição de apresentação de pistas não correspondentes. Esses dados se assemelham aos obtidos por Paracampo et al. (2001), com crianças entre 7 e 8 anos. Os autores verificaram que quando o desempenho estudado foi estabelecido por instruções, o padrão de responder manteve-se inalterado diante da mudança nas contingências. No caso do presente estudo, não se pode dizer que o desempenho dos participantes de 8 anos manteve-se inalterado quando as condições de reforço foram modificadas, 
mas verificou-se uma persistência maior no seguimento de pista atual, especialmente nas primeiras instruções não correspondentes, em comparação com as crianças de 3 e 5 anos. As crianças de 5 anos, por sua vez, apresentaram uma porcentagem intermediária de tempo de engajamento em pista atual, situando-se entre os grupos de crianças de 8 e 3 anos.

As crianças de 3 anos, apesar de se engajarem em seguimento de pista atual diante de todas as instruções não correspondentes, apresentaram a menor porcentagem média dessa categoria de respostas entre os três grupos estudados, o que indica menor persistência na conduta instruída quando esta se mostrou ineficaz para a obtenção do reforçador condicionado. Esse dado se assemelha com os obtidos por Michael e Bernstein (1991) com crianças de 4 anos e meio e 5 anos: após o estabelecimento de discriminações condicionais entre letras, formas geométricas e algarismos, e a verificação da emergência de equivalência entre os estímulos, os autores passaram a reforçar relações diferentes das estabelecidas inicialmente (por exemplo, passaram a reforçar a relação A1B2, ao invés de A1B1). Nessas condições, observou-se que o tempo que as crianças levaram para aprender as novas relações foi muito semelhante, independente do método de aquisição do desempenho inicial (por instruções, reforço diferencial ou imitação). Ou seja, houve um ajuste do responder dessas crianças às novas contingências de reforço, mesmo quando o desempenho foi adquirido por instruções.

Na presente pesquisa, o desempenho das crianças de 3 anos também se ajustou mais rapidamente às novas contingências de reforço, considerando a diminuição do tempo dispensado em seguimento de pista atual e gradual aumento de exploração do ambiente para obtenção do bloquinho de madeira. Contudo, para essas crianças mais jovens, a porcentagem média de tempo dispensado na emissão de "outros comportamentos", como olhar em direção à auxiliar de pesquisa ou ao fantoche, foi maior que para os outros dois grupos (praticamente em todas as pistas não correspondentes, à exceção de $\mathrm{C} 3 \mathrm{~m}$, na Pista 7). As crianças de 5 e 8 anos emitiram uma porcentagem baixa de "outros comportamentos", que ocorriam, em geral, nas primeiras pistas não correspondentes (com exceção de C8c e C5f).
Uma possível explicação para esse dado é que, para as crianças mais velhas, as pistas adicionais fornecidas podem ter favorecido a ocorrência de uma espécie de "variabilidade instruída", de tal forma que explorar novos locais de busca passou a ser a categoria de comportamentos mais emitida depois do abandono do seguimento de pista atual. No entanto, para as crianças de 3 anos, a presença do fantoche e da auxiliar de pesquisa, elementos que poderiam trocar o "tesouro" pelos brindes disponíveis, além de uma câmera, pode ter se constituído em variáveis sociais capazes de exercer controle sobre seu responder: ainda que o seguimento de pista atual fosse abandonado rapidamente por não se mostrar efetivo, as crianças não se engajavam na exploração do ambiente de forma imediata, sendo necessário o fornecimento de uma pista adicional (C3i e $\mathrm{C} 3 \mathrm{~m}$ ), ou de um longo tempo na emissão desses outros comportamentos até a busca do reforçador condicionado em locais não indicados pela pista atual (C3j e C31). Essa explicação parece mais plausível quando são analisadas as falas das crianças emitidas depois de não encontrarem os blocos de madeira: chamar o fantoche e dizer que não havia nada no local de busca, perguntar para a auxiliar de pesquisa o que era para fazer e assim por diante.

Variáveis sociais têm sido estudadas como determinantes da manutenção do seguimento de instruções não efetivas (Albuquerque et al., 2004; Kroger-Costa $\&$ Abreu-Rodrigues, 2012). No estudo de Monteles et al. (2006), crianças de 7 a 9 anos mantiveram o responder instruído após ele ter deixado de produzir as consequências especificadas pela instrução quando tal responder passou a produzir reforço social. Esse dado indica que, em algumas circunstâncias, consequências sociais reforçadoras podem se sobrepor à não produção dos reforçadores programados. Tal análise não parece se aplicar completamente ao presente estudo, especialmente porque, aqui, as contingências do jogo eram diversas das do estudo de Monteles et al (2006). No entanto, é preciso considerar a importância de investigações adicionais para se entender em que condições o controle exercido por variáveis sociais pode ser determinante para o comportamento instruído.

Diferenças no desempenho de crianças de diferentes faixas etárias também foram encontradas por Bentall et al. (1985). Essas diferenças parecem bastante semelhantes às encontradas no presente estudo: de- 
sempenhos mais ajustados às mudanças de contingências entre as crianças mais novas, maior persistência no comportamento instruído entre as crianças mais velhas e um desempenho intermediário entre esses dois padrões para as crianças de 5 anos. A hipótese levantada por Bentall et al., sobre interferência do repertório verbal das crianças na manutenção ou não do desempenho instruído após mudança das contingências ainda é uma questão a ser melhor investigada empiricamente, mas oferece indícios que podem orientar pesquisas futuras. Além disso, é relevante destacar que os dados aqui apresentados também sugerem que a condição inicial do jogo, em que as pistas eram correspondentes, foi suficiente para manter o seguimento inicial de pistas não correspondentes para todas as crianças de todas as idades; a manutenção desse seguimento, porém, foi mais persistente nas crianças mais velhas que nas mais novas.

É preciso considerar também que, diferente dos procedimentos geralmente empregados no estudo do controle instrucional, no presente estudo a manutenção do seguimento de instruções não correspondentes impedia a continuidade do jogo e, consequentemente, a obtenção do tesouro. Em outros procedimentos com crianças aqui descritos (Michael \& Bernstein, 1991; Monteles et al., 2006; Paracampo et al., 2001), seguir a instrução não correspondente fazia com que o participante apenas deixasse de ganhar tantos pontos quanto poderia, mas permitia a continuidade da tarefa. Além disso, é importante destacar que contingências específicas estabelecidas pelo presente procedimento, como o fornecimento de instruções adicionais, podem ter interferido nos dados produzidos. O objetivo das pistas adicionais era favorecer a emissão de respostas diferentes das especificadas pelas pistas atuais quando os participantes permanecessem muito tempo persistindo em comportamentos que não levariam ao bloquinho de madeira (seguimento de pista atual não correspondente ou outros comportamentos). A emissão "instruída" de respostas relacionadas a outros locais de busca foi reforçada e isso pode ter favorecido o controle por outros aspectos do ambiente diante de outras pistas não correspondente, além do local de busca especificado pela pista em vigor. Esse efeito pode ter obscurecido uma eventual maior persistência dos participantes no engajamento em respostas indicadas pelas pistas não correspondentes, e isso se cons- titui em uma limitação da interpretação de dados apresentada.

Apesar disso, é interessante notar que, a despeito das instruções adicionais, que poderiam ter levado todas as crianças a abandonar o seguimento de pista atual para explorar locais de busca não indicados pelas instruções não correspondentes, não foi o que ocorreu. $\mathrm{O}$ procedimento de operante livre aqui conduzido permitia aos participantes a emissão de comportamentos alternativos e potencialmente reforçadores, além de seguir ou não as instruções (desistir das pistas e buscar diretamente o tesouro, brincar com os materiais da sala, etc.). O fato de eles se engajarem no seguimento das instruções do jogo, a despeito das alternativas disponíveis e mesmo na condição de instruções não correspondentes, reafirma o estabelecimento precoce do controle por instruções (Catania, 1999; Veiga et al., 2012).

Tomadas em conjunto, as variáveis aqui discutidas reafirmam a multideterminação de operantes instruídos, bem como a força do controle por instruções (Albuquerque, 2005), mesmo em crianças muito novas. Os resultados do presente estudo também evidenciam a importância de se aprofundar o estudo sobre a gênese do controle por instruções, uma vez que o estabelecimento desse repertório é de fundamental importância para a cultura. Apenas afirmar que o comportamento instruído é "insensível” às contingências parece ser uma descrição pouco acurada do processo; o direcionamento das pesquisas sobre o tema devem se concentrar, sim, em determinar as condições sob as quais o responder se mantém ou se altera, a despeito da mudança nas contingências.

\section{REFERÊNCIAS}

Albuquerque, L. C. (2005). Regras como instrumento de análise do comportamento. In L. C. Albuquerque (Org.), Estudos do comportamento (pp. 143-176). Belém: Edufpa.

Albuquerque, L. C., de Souza, D. G., Matos, M. A., \& Paracampo, C. C. P. (2003). Análise dos efeitos de histórias experimentais sobre o seguimento subseqüente de regras. Acta Comportamentalia, 11, 87-126.

Albuquerque, L. C., Matos, M. A., de Souza, D. G., \& Paracampo, C. C. P. (2004). Investigação do controle por regras e do controle por histórias de reforço sobre o comportamento humano. Psicologia: Reflexão e Crítica, $17,395-412$. 
Albuquerque, N. M. A., Paracampo, C. C. P., \& Albuquerque, L. C. (2004). Análise do papel de variáveis sociais e de consequências programadas no seguimento de instruções. Psicologia: Reflexão e Crítica, 17, 31-42.

Albuquerque, L. C., Reis, A. A., \& Paracampo, C. C. P. (2008). Efeitos de histórias de reforço, curtas e prolongadas, sobre o seguimento de regras. Acta Comportamentalia, 16, 305-332.

Baron, A., \& Galizio, M. (1983). Instructional control of human operant behavior. The Psychological Record, 33, 495-520.

Baxter, G. A., \& Schlinger, H. (1990). Performance of children under a multiple random-ratio random-interval schedule of reinforcement. Journal of the Experimental Analysis of Behavior, 54, 263-271.

Bentall, R. P., Lowe, C. F., \& Beasty, A. (1985). The role of verbal behavior in human learning: II. Developmental differences. Journal of the Experimental Analysis of Behavior, 43, 165-181.

Catania, A. C. (1999). Aprendizagem: Comportamento, linguagem e cognição. Porto Alegre: Artes Médicas Sul.

Catania, A. C., Matthews, B. A., \& Shimoff, E. (1982). Instructed versus shaped human verbal behavior: Interactions with nonverbal responding. Journal of the Experimental Analysis of Behavior, 38, 233-248.

Catania, A. C., Shimoff, E., \& Matthews, B. A. (1989). An experimental analysis of rule-governed behavior. In S. C. Hayes (Org.), Rule-governed behavior: Cognition, contingencies, and instructional control (pp. 119-150). New York: Plenum.

Hayes, S. C., Brownstein, A. J., Zettle, R. D., Rosenfarb, I., \& Korn, Z. (1986). Rule governed behavior and sensitivity to changing consequences of responding. Journal of the Experimental Analysis of Behavior, 45, 237-256.

Joyce, J. H., \& Chase, P. N. (1990). Effects of response variability on the sensivity of rule-governed behavior. Journal of the Experimental Analysis of Behavior, 54, 251-262.

Kroger-Costa, A., \& Abreu-Rodrigues, J. (2012). Effects of historical and social variables on instruction following. The Psychological Record, 62, 691-706.

Lowe, C. F., Harzem, P., \& Bagshaw, M. (1978). Species differences in temporal control of behavior II: Human performance. Journal of the Experimental Analysis of Behavior, 29, 351-361.

Keller, F. S., \& Schoenfeld, W. N. (1996). Principles of psychology. B.F. Skinner Foundation. Copley Publishing Group. (Trabalho original publicado em 1950.)

Madden, G. J., Chase, P. N., \& Joyce, J. H. (1998). Making sense of sensitivity in the human operant literature. The Behavior Analyst, 21, 1-12.

Matos, M. A. (2001). Comportamento governado por regras. Revista Brasileira de Terapia Comportamental e Cognitiva, 3, 51-66.
Matthews, B. A., Shimoff, E., Catania, A. C., \& Sagvolden, T. (1977). Uninstructed human responding: Sensitivity to ratio and interval contingencies. Journal of the Experimental Analysis of Behavior, 27, 453- 467.

Michael, R. L., \& Bernstein, D. J. (1991). Transient effects of acquisition history on generalization in a matching-tosample task. Journal of the Experimental Analysis of Behavior, 56, 155-166.

Monteles, K. M. C., Paracampo, C. C. P., \& Albuquerque, L. C. (2006). Efeitos de uma história de reforço contínuo e de consequiências sociais sobre o seguir regras. Psicologia: Reflexão e Crítica, 19, 186-196.

Nico, Y. (1999). Regras e insensibilidade: conceitos básicos, algumas considerações teóricas e empíricas. In R. R. Kerbauy \& R. C. Wielenska (Orgs.), Sobre Comportamento e Cognição: psicologia comportamental e cognitiva: da reflexão teórica à diversidade da aplicação (pp. 31-39). Santo André: ESETec Editores Associados.

Paracampo, C. C. P., \& Albuquerque, L. C. (2004). Análise do papel das consequiências programadas no seguimento de regras. Interação em Psicologia, 8, 237-245.

Paracampo, C. C. P., \& Albuquerque, L. C. (2005). Comportamento controlado por regras: revisão crítica de proposições conceituais e resultados experimentais. Interação em Psicologia, 9, 227-237.

Paracampo, C. C., Albuquerque, L. C., Cavalló, B. N., \& Torres, S. M. (2009). Análise do controle por regras apresentadas em histórias infantis. Revista Brasileira de Análise do Comportamento, 5(2), 107-122.

Paracampo, C. C. P., Souza, D. G., Matos, M. A., \& Albuquerque, L. C. (2001). Efeitos de mudança em contingências de reforço sobre o comportamento verbal e não verbal. Acta Comportamentalia, 9, 31-55.

Perone, M. (1991). Experimental design in the analysis of free-operant behavior. In I. H. Iversen \& K. A. Lattal (Eds.), Techniques in the behavioral and neural sciences: Vol. 6. Experimental analysis of behavior, Part 1 (pp. 135-171). Amsterdam: Elsevier.

Shimoff, E., Catania, A. C., \& Matthews, B. A. (1981). Uninstructed human responding: Sensitivity of low-rate performance to schedule contingencies. Journal of the Experimental Analysis of Behavior, 36, 207-220.

Shimoff, E., Matthews, B. A., \& Catania, A. C. (1986). Human operant performance: Sensitivity and pseudosensitivity to contingencies. Journal of the Experimental Analysis of Behavior, 46, 149-157.

Skinner, B. F. (1957). Verbal Behavior. New York: Appleton-Century-Crofts.

Skinner, B. F. (1969). An operant analysis of problem solving. In B. F. Skinner (Org.), Contingencies of reinforcement: A theoretical analysis (pp. 133-171). New York, NY: Appleton-Century-Crofts. (Trabalho original publicado em 1966) 
Skinner, B. F. (1974). About Behaviorism. London: Penguin.

Teixeira Júnior, R. R. (2009). Variáveis do comportamento governado por regras: Uma análise de estudos da área. Acta Comportamentalia, 17, 351-385.

Teixeira Júnior, R. R., \& Paracampo, C. C. P. (2010). Efeitos de instruções preliminares, específicas e mínima sobre uma tarefa experimental e seguimento de instruções. Revista Brasileira de Análise do Comportamento, 6, 89104.

Teixeira Júnior, R. R., \& Paracampo, C. C. P. (2012). Efeitos de instruções preliminares sobre o seguimento de regras. Psicologia: Teoria e Pesquisa, 28, 267-274.

Thomaz, C. R. C., \& Nico, Y. C. (2007). Quando o verbal é insuficiente: Possibilidades e limites da atuação clínica dentro e fora do consultório. In D. R. Zamignani, R. Kovac, \& J. S. Vermes (Eds.), A clínica de portas abertas: Experiências e fundamentação do acompanhamento terapêutico e da prática clínica em ambiente extraconsultório (pp. 47-75). Santo André, SP: Esetec/Paradigma.

Veiga, D. I., Schmidt, A., \& Biscouto, K. D. (2012). Uma análise das funções de instruções em um procedimento de operante livre com crianças. Acta Comportamentalia, 20, 45-69.
Velasco, S. M., Garcia-Mijares, M., \& Tomanari, G. Y. (2011). Fundamentos metodológicos da pesquisa em análise experimental do comportamento. Psicologia em Pesquisa, 4(2), 105-155.

Weiner, H. (1964). Response cost and fixed-ratio performance. Journal of the Experimental Analysis of Behavior, 7, 79-81.

Weiner, H. (1969). Controlling human fixed-interval performance. Journal of the Experimental Analysis of Behavior, 12, 349-373.

Weiner, H. (1970). Instructional control of human operant responding during extinction following fixed-ratio conditioning. Journal of the Experimental Analysis of Behavior, 13, 391-394.

\section{Nota:}

Recebido em: 09/10/2012

Última revisão em: 29/06/2013

Aceito em: 01/08/2013

1 A redação deste manuscrito foi apoiada pelo Instituto Nacional de Ciência e Tecnologia sobre Comportamento, Cognição e Ensino - INCT-ECCE (CNPq, processo 573972/2008-7 e FAPESP, processo 2008/57705-8). 